\title{
COMPARING VIRTUAL REALITY AND DESKTOP INTERFACE FOR REVIEWING 3D CAD MODELS
}

\author{
Horvat, Nikola (1); Škec, Stanko (1,2); Martinec, Tomislav (1); Lukačević, Fanika (1); Perišić, \\ Marija Majda (1) \\ 1: University of Zagreb; 2: Technical University of Denmark
}

\begin{abstract}
Use of virtual reality (VR) is considered beneficial for reviewing 3D models throughout product design. However, research on its usability in the design field is still explorative, and previous studies are often contradictory regarding the usability of VR for 3D model review. This paper argues that the usability of VR should be assessed by analysing human factors such as spatial perception and taking into consideration the complexity of the reviewed product. Hence, a comparative evaluation study has been conducted to assess spatial perception in desktop interface-based and VR-based review of 3D models of products with different levels of complexity. The results show that participants in VR more could perceive the fit of user interface elements, and estimation of the model dimensions had a lower relative error than in desktop interface. It has been found that various sensory cues are used to perceive the model size and that the employed sensory cues depend on the level of complexity. Finally, it is proposed that differences between a desktop interface and VR for reviewing models are more evident when reviewing models of higher complexity levels.
\end{abstract}

Keywords: Virtual Engineering (VE), Visualisation, Computer Aided Design (CAD), Spatial Perception, Product modelling / models

Contact:

Horvat, Nikola

University of Zagreb, FSB

Department of Design

Croatia

nikola.horvat@fsb.hr

Cite this article: Horvat, N., Škec, S., Martinec, T., Lukačević, F., Perišić, M.M. (2019) 'Comparing Virtual Reality and Desktop Interface for Reviewing 3D CAD Models', in Proceedings of the 22nd International Conference on Engineering Design (ICED19), Delft, The Netherlands, 5-8 August 2019. DOI:10.1017/dsi.2019.198 


\section{INTRODUCTION}

In the detailed design phase of product design, 3D computer-aided design (CAD) models are usually used as a representation of a product (Chandrasegaran et al., 2013). At a minimum, they represent a 3D form of a product with some minor simplifications (e.g. threads) and provide a better understanding of the design when compared to 2D models (Hou and Hollands, 2009).

3D CAD models are commonly visualised via a desktop interface (DI). However, the use of more immersive technology, where users can navigate and interact with the model in 3D, might be beneficial for an understanding of the product. For example, mediating a design review process with the use of virtual reality (VR) might result in a higher number of identified design issues (Banerjee et al., 2002; Satter and Butler, 2015; Berg and Vance, 2016; Freeman et al., 2018). The use of VR reduces the required cognitive effort needed for envisioning the product and, therefore, enables a more thorough analysis of a product (Liu et al., 2014). In contrast, some studies reported that the review of 3D CAD models in VR resulted in the same or even lower number of identified issues (Hou and Hollands, 2009; de Casenave and Lugo, 2017). Hence, it is still unclear when to implement VR over DI for reviewing 3D CAD models (de Casenave and Lugo, 2017). The reason might be due to the researchers' interest on the effect of VR review outcomes (i.e. the number of issues detected, time to conduct a task) rather than on the human factors such as spatial perception (Paes et al., 2017). Developing understanding of the influence that different technologies have on spatial perception will enable identification of parameter values upon which those technologies would be most efficient at simulating reality and facilitating the design process (Paes et al., 2017).

While comparing VR and other technologies for reviewing CAD models, researchers in the engineering design field pointed out that simplicity/complexity of the observed product might influence the study outcomes (de Casenave and Lugo, 2017; Freeman et al., 2018). Previous studies on the usability of VR have ranged from geometric primitives to more complex devices such as cars in engineering design domain (Berg and Vance, 2016; de Casenave and Lugo, 2017; Freeman et al., 2018). Furthermore, other fields such as construction and architectural design have also tackled the usability of VR for CAD model reviews. Within these domains, products are usually larger structures (e.g. buildings) which would, according to the Theory of technical systems (Hubka and Eder, 1988), fall into the highest complexity category. However, none of the identified studies has explored to what extent the spatial perception in VR differs for products of different complexity levels.

Since comparison can introduce an element of more objective appraisal, this research compares DI and VR regarding spatial perception for reviewing 3D CAD models. More precisely, this research aims to answer two research questions:

- How does spatial perception differ for DI-based and VR-based review of 3D CAD models?

- Does spatial perception using DI-based and VR-based review of 3D CAD models change with the products' complexity level?

\section{BACKGROUND}

\subsection{Visualisation of products in engineering design}

When observing external representations, such as CAD models, the mental imagery in humans' brain depends on the type of representation used. In general, common types of representing a form of the products are 2D and 3D models (Chandrasegaran et al., 2013). When using 2D models to describe the geometry of a 3D object, convention, codes and standards are used to ensure unambiguousness of the representation. Perceiving 3D geometry from two-dimensional representation requires technical knowledge and high spatial reasoning skills. On the other hand, 3D models present products without excluding features, except in cases where complete representation is computationally demanding (e.g. threads). Thus, the transformation of product representation into 3D space is performed by the computer, whereas designers can direct their cognitive load at a more detailed analysis of the product (Liu et al., 2014). In the same line of research, Hou and Hollands (2009) reported that reviewing 3D CAD models on DI is easier and increases the number of found issues when compared to reviewing 2D models.

Product geometry reviews are often performed using 3D CAD models (Freeman et al., 2018). Their visualisation can be through a 2D projection of a 3D scene on a DI or as higher fidelity 3D representation using VR technology. Currently, most of the VR technologies can be divided into Cave Automatic 
Virtual Experience (CAVE) and Head Mounted Display (HMD) systems. CAVE systems are based on projecting image on screens inside the virtual room and require substantial investment capital. On the other hand, the more affordable HMD systems utilise two small screens placed near the eyes of the user. Based on recent findings (Donalek et al., 2014), the usage of VR technologies can lead to a better perception of geometry and more intuitive data understanding than DIs. Current VR systems allow navigation of avatars and manipulation with 3D CAD models, thus enabling interaction while reviewing the CAD model in a VR environment. Even though VR offers a more natural representation of 3D CAD models, DIs are still more common for design reviews. However, the recent proliferation of new low-cost VR technologies has the potential to increase the usage of VR in reviewing 3D CAD models (Coburn et al., 2017).

\subsection{VR in reviewing CAD models}

Studies regarding the usability of the VR in engineering design field have explored the tangible aspects of the review process (e.g. time), various design review aspects (e.g. the number of identified issues), and analysis of human factors (e.g. spatial perception).

By observing the time needed to conduct navigation and error finding in DI and VR environment, Satter and Butler (2015) found that participants in a VR environment required significantly less time to perform the given task. On the other hand, de Casenave and Lugo (2017) reported that navigation and manipulation tasks take more time in VR environment. They stated that observation of more features in a VR environment results in a longer duration of design reviews (de Casenave and Lugo, 2017).

Hou and Hollands (2009) argued that VR systems have a potential for reviewing CAD models since they preserve realistic characteristics of a product, provide natural feeling when interacting with the model, allow users to develop precise spatial understanding, and produce a better sense of presence. Liu et al. (2014) conducted a questionnaire on the usage of VR in which participants reported that VR helped them to understand the design better and that they felt more confident about their perception. In Berg and Vance's (2016) case study of design teams, participants stated that VR environment helped them in keeping attention on the design review since no visual distractions from outer world could interrupt the process. In de Casenave and Lugo's (2017) experiment, participants were instructed to identify errors in a product assembly, and the results suggest the same error finding performance in VR and DI. Freeman et al. (2018) observed VR with basic (e.g. pan, rotate, scale) and advanced options (e.g. hiding, using exploded view, parametric model update). They found that having advanced options (e.g. hiding components) improves understanding of designs in terms of counting the number of gears in a gearbox. Use of the advanced options boosted the confidence of the participants regarding answers related to design understanding (Freeman et al., 2018).

Studies of VR systems related to the analysis of human factors are often focused on the different aspects of spatial perception. Analysing depth perception for the cases where users reviewed 3D CAD models in DI and VR environment, Gîrbacia et al. (2012) concluded that participants performed better in the VR environment. Satter and Butler (2015) observed spatial awareness by measuring the distance between a perceived and actual location of a 3D cube and reported an improvement of the spatial awareness in VR. Participants in Berg and Vance's (2016) study stated that VR improved their sense of the spatial relationships between product components. In another study, the same researchers stated that spatial relationships are easily addressed in VR (Berg and Vance, 2017). In the architectural design field, Paes et al. (2017) compared VR and DI representations of a building model and found that the use of VR improves spatial perception. Researchers also suggested that the benefits of VR might change with the complexity of the observed model. For example, Wolfartsberger et al. (2017) argue that, since VR mitigates the spatial constraints, VR systems can be applied for reviewing large products. On the other hand, models with a lower level of complexity might diminish the advantages of VR in identifying issues (de Casenave and Lugo, 2017). Freeman et al. (2018) reported that models with a higher level of complexity might be more beneficial to review with the advanced VR options than lower complexity models. However, none of the reviewed studies has investigated or compared the applicability of VR for reviewing products of different complexity levels.

In the abovementioned studies, researchers used different metrics and often did not discuss the results to explain and support their research findings. It is assumed that spatial perception is enhanced with the use of VR, but very few studies have been conducted to find empirical evidence that would support this claim. Furthermore, the level of product complexity might moderate the effect of VR on the reviewing process, and, as such, this phenomenon will be investigated within this study. 


\section{METHODOLOGY}

A repeated measures experiment was used to mitigate previously identified issues from the literature. User responses were collected using qualitative data gathering methods (i.e. semi-structured interviews and observations) and further analysed by the thematic coding procedure. The applied methodological framework is based on previously reported studies on comparative evaluation of VR and DI (Paes et al., 2017). The study was conducted in seven steps: 1) Development of experimental procedure and interview questions, 2) Identification of 3D CAD models; 3) Development of experimental layout 4) Carrying out the pilot experiment; 5) Selection of study participants 6) Running the experiments; 7) Thematic coding analysis. Section 3.1 outlines the experiment preparation (steps 1-5), while the experimental procedure is described in Section 3.2.

\subsection{Experiment preparation and layout}

The selection of the 3D CAD models used in the experiment was based on the complexity classification given in Theory of technical systems (Hubka and Eder, 1988): component, mechanism, device, and complex machine (CM) unit. The models of the component and CM unit level were the same in both environments while the models for the mechanism and device level had the same functionality but different dimensions and structure. The overview of the models, the dimensions and the allocated review time are shown in Figure 1.

\begin{tabular}{|l|l|l|l|l|}
\hline & Level 1: Component & Level 2: Mechanism & Level 3: Device & Level 4: Complex machine unit \\
\hline $\begin{array}{l}\text { Desktop interface } \\
\text { (DI) environment }\end{array}$ & & \\
\hline $\begin{array}{l}\text { Virtual reality (VR) } \\
\text { environment }\end{array}$ & &
\end{tabular}

Figure 1. 3D CAD models used for the experimentation.

The experimental layout which includes necessary hardware is shown in Figure 2. For reviewing CAD models via DI, SolidWorks CAD software was used. Models were presented on a 21.5" monitor with the resolution of 1920x1080, and they could be navigated with the use of traditional mouse and keyboard. Participants used HTC Vive VR system to review CAD models within the VR environment. The VR system consisted of an HMD display, two controllers and base stations for capturing movement.

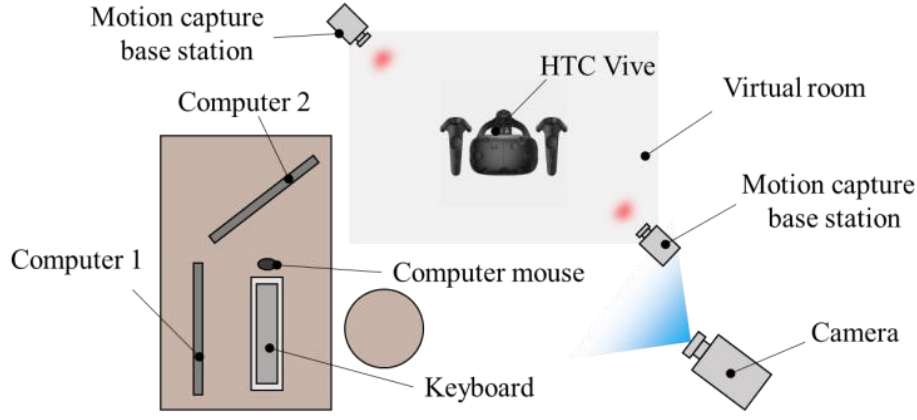

Figure 2. Experimental layout.

The CAD model was displayed in the 3DExperience platform by utilising the incorporated VR tools. These tools allow movement of avatar around the model, screenshot capture, and translation and rotation of CAD models using the controllers. Other advanced functions such as hiding of parts and making section cuts were not available directly from the VR environment. However, a third party could activate the functions without interrupting the review process. Besides interacting with the 3D CAD model, users could navigate their avatar in the VR environment by walking around the virtual room (size $2 \mathrm{~m} \times 1.5 \mathrm{~m}$ ) 
or by using controllers. In order to test the equipment, validate the experimental procedure and assess the time required for reviewing each model, a pilot study was conducted. In total 15 participants were selected from a pool of third and fourth-year students of mechanical engineering which had previous experience in CAD modelling. All of the participants had passed the basic course on CAD modelling where they use constructive solid geometry technique to model components and build assemblies in SolidWorks CAD software.

\subsection{Experimental procedure}

Experimental procedure consisted of five steps. Firstly, an initial semi-structured interview was conducted to gather information on participants' experience with CAD and VR. The examples of interview questions are given in Table 1. Secondly, a review of the 3D CAD models of varying complexities (from lowest to highest complexity) was conducted in DI. The participants were familiar with the mouse and keyboard manipulation functions in SolidWorks software, so the training was omitted from the procedure. In the third step, participants were given training on the usage of VR equipment required for conducting a review. Participants were instructed to move to the borders of the virtual room and were given a test model of a product with the component level of complexity (different than the one used for reviewing). When presented with the test model in the VR environment, participants were instructed to go through the basic functions: translation and rotation of the avatar using controllers and physical movement, translation and rotation of models around all axes, and screenshot capture. The training was followed by an acclimation period until the participants felt comfortable to start reviewing the models in the VR environment. After the preparation and acclimation period, participants reviewed 3D CAD models of varying complexity levels (from lowest to highest) in the VR environment. While reviewing the models, participants were asked questions regarding dimension estimation, model details, ergonomic factors etc. (Table 1). However, as this paper aims to compare spatial perception in two observed environments, irrelevant questions were excluded from the Results section. As a final step, a post-experiment interview was conducted to capture participants' well-being, experience regarding representation quality, immersion and spatial perception, and views on capabilities of the two systems used for reviewing models (Table 1).

The entire experiment, except for one post-experimental interview (technology issues), was recorded to prevent information loss. The insights from the non-recorded post-experiment interview are, therefore, based only on the notes taken by the interviewer.

Table 1. Questions asked during the initial interview, review, and post-experiment interview.

\begin{tabular}{|l|l|}
\hline & Sample Questions \\
\hline $\begin{array}{l}\text { Initial } \\
\text { interview }\end{array}$ & $\begin{array}{l}\text { - Do you have any experience in using VR tools? } \\
\text { - How much experience do you have with the use of DI? }\end{array}$ \\
\hline $\begin{array}{l}\text { Review } \\
\text { questions }\end{array}$ & $\begin{array}{l}\text { - Can you estimate the dimensions of the model? } \\
\text { - If yes, can you provide the dimension values? } \\
\text { - What do you think about the dimension fit of the user interface elements of the reviewed model? }\end{array}$ \\
\hline $\begin{array}{l}\text { Post- } \\
\text { experiment } \\
\text { interview }\end{array}$ & $\begin{array}{l}\text { - Do you believe that VR helped you in understanding the model size and getting a spatial perception? } \\
\text { - Did you have enough time for reviewing models in both environments? }\end{array}$ \\
\hline
\end{tabular}

\section{RESULTS}

In this section, results of the experimental study were aggregated across all participants. Section 4.1 presents results of the initial interview which focuses on participants' prior experience. Sections 4.2-4.4 present findings from reviewing models of different complexity in terms of perceiving the model size, perceiving the user interface elements (UIE) of the model, and estimating dimensions. The last section presents results of the post-experiment interview which give insights into participants' review experiences.

\subsection{Initial interview}

Majority of participants $(n=13)$ have had experience in more than one CAD software and have previously designed a technical system of at least the second level of complexity. Most of the participants had either no experience in using VR system $(n=8)$ or only short experience of up to 10 minutes $(n=6)$, while one participant experienced 3-4 hours of playing VR computer games. 


\subsection{Perceiving the model size}

Participants' answers on the ability to perceive the size of the model were aggregated regarding the four complexity levels and two environments (Figure 3).

In the DI, none of the participants could perceive the size of the component, and several participants expressed the need for a reference $(n=3)$ or requested additional information regarding the component functionality $(n=1)$. At the second complexity level, the majority $(n=9)$ of the participants were not able to perceive the size, out of which three pointed out the need for a reference. Others could perceive the size of the mechanism $(n=6)$ explaining that they either have previous experience with clutches $(n=4)$ or that they based their answer on the UIE of the model $(n=2)$. At the third complexity level, most of the participants could perceive the size $(n=14)$ by observing the UIE $(n=9)$ or by previous experience with scooters $(n=1)$. Finally, all of the participants could perceive the size of the complex machine unit $(n=15)$, out of which some pointed out the UIE as a reference $(n=7)$ or previous experience regarding the hydraulic press $(\mathrm{n}=1)$.

In the VR, participants could perceive the size of the models regardless of the complexity level. At the first complexity level, only the controllers were mentioned as the support in perceiving the model size $(\mathrm{n}=1)$. At the second complexity level, participants perceived the model size using controllers $(\mathrm{n}=5)$, a combination of controllers and themselves $(n=4)$, or only themselves $(n=2)$ as a reference. At the third complexity level, three participants stated the use of just controllers for perceiving the size, six participants reported the use of themselves and controllers, and three participants reported the use of themselves only. Finally, at the highest complexity level, only one participant reported using controllers for perceiving the model size, while others stated the use of controllers and themselves $(n=2)$, or use of themselves only $(n=4)$. Use of controllers and participants' senses is shown in Figure 6.

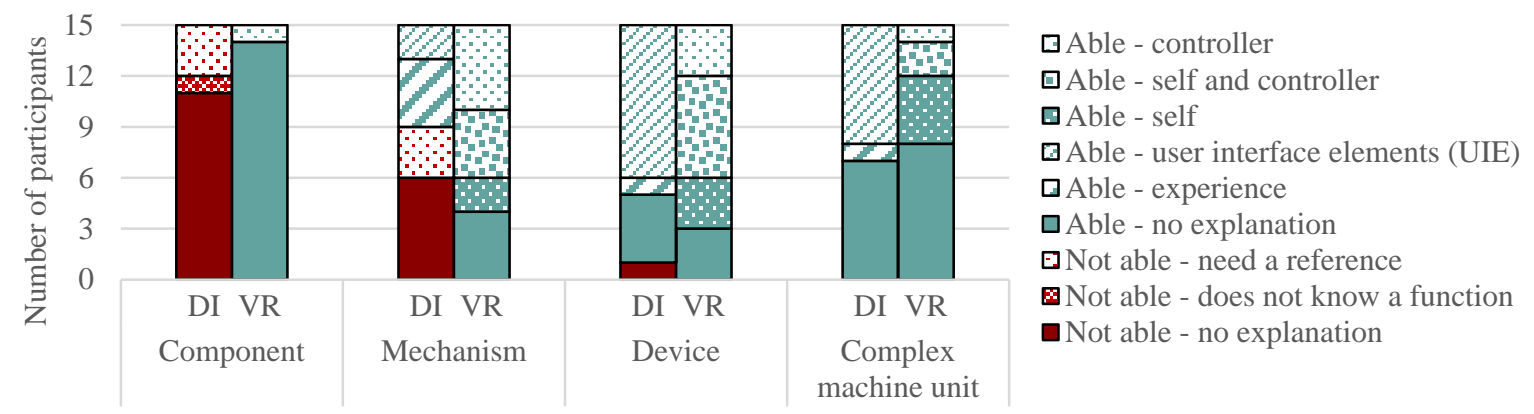

Figure 3. Ability to perceive the model size in two environments.

\subsection{Estimating dimensions of the models}

The relative error of participants' dimension estimations was used as an objective measure of the difference in spatial perception between the two environments. The boxplots in Figure 4 represent the relative error for models of different complexity levels. As reported in Section 4.2, participants could not perceive the model size in some cases (Figure 3), so they were provided with a reference dimension to facilitate their estimation in DI for the first two complexity levels. The given reference dimensions, as well as the estimated dimensions, are outlined in Figure 1.

On average, estimating dimensions of the component (1 - Height and 1 - Diameter) with a given reference (1 - Length) in DI resulted in dimension overestimation with a mean relative error of 0.5 for the height and 0.4 for the diameter. At the second complexity level, participants were also given reference dimension ( 2 - Length), and they estimated height ( 2 - Height) with a mean relative error of 0.02 , and diameter ( 2 - Diameter) with a mean relative error of -0.04 . At the highest complexity level, participants were able to perceive the size of the model (Figure 3) and were therefore asked to estimate dimensions without a reference. Participants reported length (4 - Length) of the hydraulic press with a relative error ranging from -0.31 to 0.89 with a mean relative error of 0.16 . Results for the reported height (4 - Height) show an overestimation of the dimension with a relative error ranging from 0 to 1.02 and a mean value of 0.31 .

In the VR, participants reported three dimensions of the clutch, namely height (2 - Height), length (2 Length) and diameter ( 2 - Diameter). The height of the clutch was estimated by 11 participants with the relative error ranging from -0.23 to 1.19 and a mean value of 0.18 . Length was underestimated with the relative error ranging from -0.4 to 0.04 and a mean value of -0.22 . The diameter of the clutch estimation error ranged from -0.38 to 0.56 and a mean value of -0.05 . At the highest complexity level, participants 
underestimated length ( 4 - Length) of the hydraulic press with the relative error ranging from -0.42 to 0.03 and a mean value of -0.13 . The height of the hydraulic press (4 - Height) was estimated most accurately with the relative error ranging from -0.04 to 0.01 and a mean relative error of 0 .
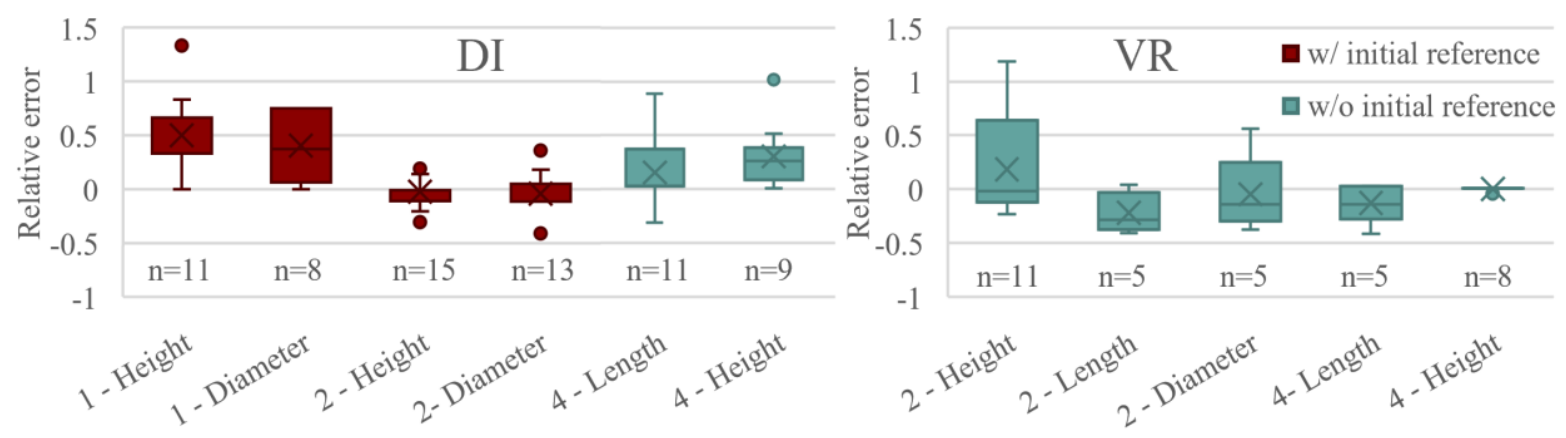

Figure 4. Estimated dimensions; see Figure 1 for description of dimensions.

\subsection{Perceiving the fit of user interface elements in CAD models}

User interface elements (UIE) are here defined as components or component features that the user of the technical system interacts with. Participants' answers on the UIE fit of CAD models were categorised according to the environment and the complexity level. Since the component model did not have any UIE, no data regarding the UIE fit at the lowest complexity level was gathered. On the other hand, models at other complexity levels had one or more UIEs, and the data regarding UIE fit were categorised based on the name of the UIE. Figure 5 depicts the participants' answers regarding the UIE fit.

In the DI, regardless of the complexity level, some participants could not perceive the fit of a UIE. At the second complexity level, nine participants could perceive the fit of UIE (i.e. handle ball), but no agreement was found on perceived fit: four participants thought that the handle ball was of the right size $(n=4)$, two reported that it was too big, and three said it was too small. At the third complexity level, two participants could not perceive the distance between seat and steering bar. Six participants who could estimate the UIE fit reported that the steering bar is either too close $(n=1)$ or too far from $(n=5)$ the seat. Regarding steering bar width, five participants could not perceive the UIE fit; one participant reported that the steering bar is of the adequate width, and one stated that the width of hands on the steering bar is too large. At the highest complexity level, two participants could not report on the height fit of a control panel, and three participants could not perceive if the distance between the robot and table is enough to pass through. Perceiving the height of the control panel, participants reported that the control panel is either high enough $(n=4)$ or is too low $(n=1)$. When it comes to the distance between the table and robot, six participants reported that they could pass through the gap, while three participants stated that the passage is too narrow.

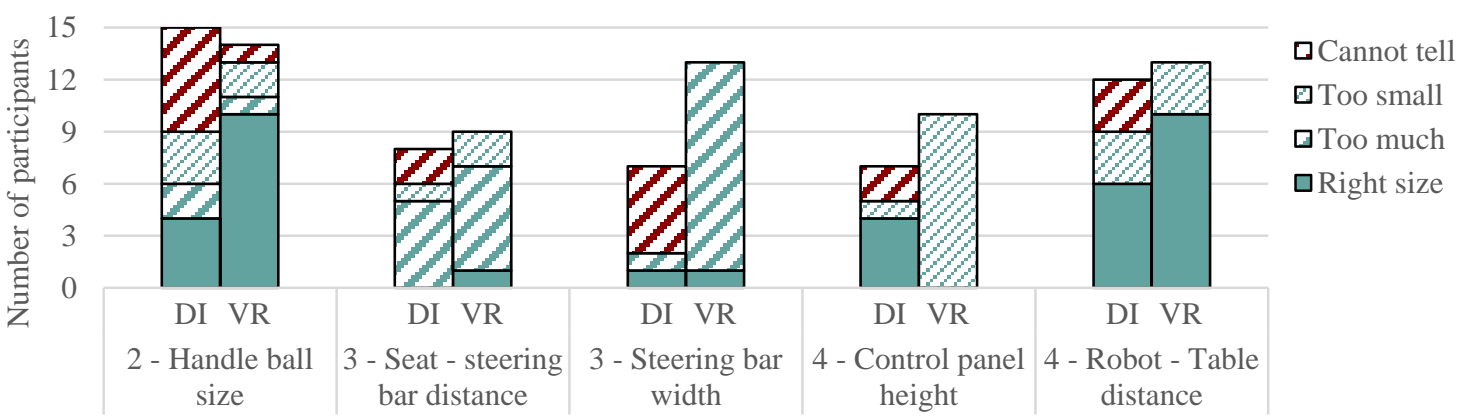

Figure 5. Comparison of UIE fit perception within the two environments.

Within VR, participants could perceive the fit of all UIEs, except for the one participant who could not report on the fit of the handle ball. At the second complexity level, the majority $(n=10)$ of the participants reported that the UIE fits their hand adequately. Only one participant perceived the handle ball as too big, and two said that it is too small. When reviewing the UIE of the scooter, most of the participants $(n=6)$ perceived that the steering bar and seat are too far from each other. One participant thought that the distance is appropriate, while two of the participants reported that the steering bar and the seat are too close to each other. The width of the steering bar was perceived as adequate by one 
participant, while others agreed that it was too wide $(n=12)$. At the highest complexity levels, all of the participants stated that the control panel is too low $(n=10)$. Ten participants reported that the distance between the robot and the table was wide enough to pass through, while the remaining participants $(n=3)$ thought that the gap is not wide enough. Figure 6 shows some examples of perceiving the UIE fit in the VR environment.

\subsection{Post-experiment interview}

Participants confirmed that they had enough time to answer questions while reviewing models. Most of the participants $(n=9)$ stated that, even though they could perceive the model size with a given reference dimension, they were more certain about its dimensions in VR. Participants also noted that it was easier to estimate dimensions in a VR environment $(n=13)$. Finally, participants pointed out that the main disadvantages of the VR system over DI were picture quality $(n=8)$ and the need for the adaptation to the system $(\mathrm{n}=2)$.
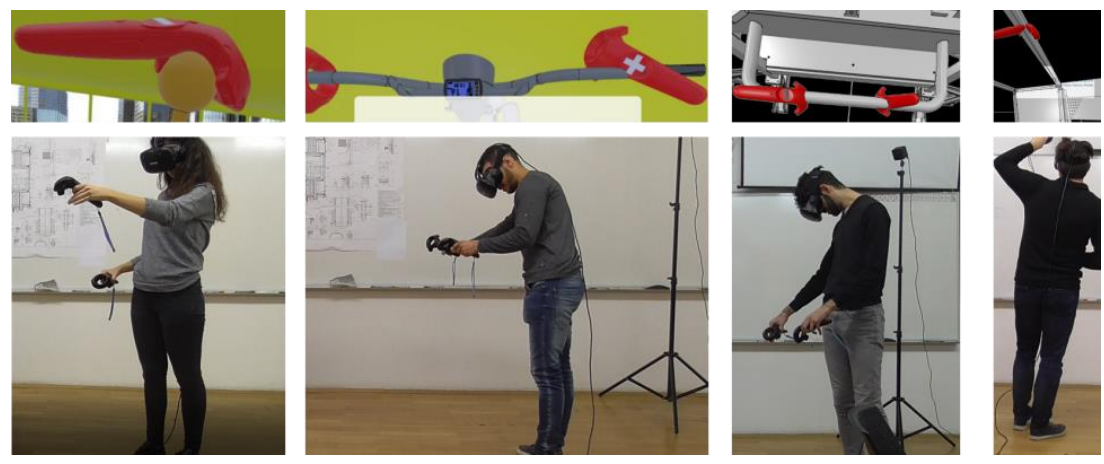

Figure 6. Using controllers and sensory cues while reviewing 3D models in VR environment.

\section{DISCUSSION}

In this section, the results and the limitations of the experimental study are discussed. In Section 5.1, results have been aggregated across all four levels of complexity in order to provide overall insights on spatial perception within the two environments. Section 5.2 reflects on the differences in spatial perception which have been identified across different complexity levels. Finally, Section 5.3 presents the limitations of the research.

\subsection{Comparison of spatial perception in DI and VR environments}

Results related to the DI environment suggest that participants could estimate dimensions of the model if they were familiar with its function, or if they were able to identify the model's UIE. This finding is in line with previous studies which have argued that experience influences spatial perception in DI (Paes et al., 2017). Furthermore, once participants identified an initial reference via the DI (either on their own or provided by the experimenter), a majority of them could perceive the model size and hence estimate its dimensions (Figure 4) and report on the UIE fit (Figure 5). On the other hand, in a VR environment, participants could have utilised controllers and their proprioceptive sense, thus enhancing spatial perception regardless of the function of the reviewed technical system. Additionally, the consistent scale of the model might have facilitated spatial perception in VR. These findings suggest that humans use various sensory cues to get the spatial perception of an object (Mohler et al., 2013), whereas the absence of sensory cues lowers the level of spatial perception as indicated by the overall DI and VR differences. Indeed, when immersed in VR, the participants were more certain about the model dimensions as reported in the post-experiment interview. These claims are in line with the results reported in Figures 3 and 5, where it is evident that the participants had no problems estimating the dimensions and UIE fit within the VR environment (as opposed to the results for the DI, where some participants could not provide such estimations). The results on dimension estimations provide additional support for the argument of better spatial perception within VR as opposed to DI, particularly at the highest complexity level, where no initial reference was given. However, the results indicate better spatial perception when using DI compared to VR, in cases when participants were given initial reference dimensions (small differences in reported dimension estimations at the second complexity level example in Figure 4). Although the relative error for the clutch in VR seems high, there are few possible explanations for the 
imprecise dimension estimations. First, as indicated by two participants, they found it difficult to estimate absolute dimensions in general. Previous studies have found that users usually underestimate dimensions in both real and virtual environment (see 2 - Length, 2- Height, and 4 - Length in Figure 4 VR) with distances in a virtual environment being less accurate than distances in a real environment (Gooch and Willemsen, 2002). Second, it might be that the inability of seeing their own body influenced correctness of the dimensions estimated by the participants since they could only use their height and controller positions as references in VR. Finally, it might be that the absolute values of the estimated dimensions influenced the results. Nevertheless, this study supports previous findings of better spatial perception in VR in comparison to DI (Paes et al., 2017).

\subsection{Comparing spatial perception in DI and VR for different complexity levels}

The results regarding the participants' ability to perceive model size provide insights on the effects that the two environments have on spatial perception at different levels of model complexity. Figure 3 shows that the increase in the level of complexity implies an increase in the number of participants which could perceive the model size in DI. The possible explanation might be the increased likelihood that the reviewer will recognise a part in the higher complexity model and use it as an initial reference. Another explanation might be that more complex devices have more UIEs which could help the reviewer in perceiving the model size. Moreover, as already reported in previous studies (de Casenave and Lugo, 2017; Freeman et al., 2018), the change in complexity of the reviewed technical systems within VR also suggest an effect on spatial perception. Particularly, the experiment participants rarely reported the use of proprioceptive sense as a reference when observing lower complexity models while for the model of highest complexity, the proprioceptive sense was used by most of the participants.

Furthermore, since participants indicated fewer references while reviewing 3D models with a lower level of complexity in VR, it can be argued that reviewing these models utilises different sensory cues in comparison to reviewing the more complex models. Indeed, the results of the dimension estimation in VR suggest better spatial perception for higher complexity levels, indicating that VR performs better than DI in supporting the sensory cues necessary for perceiving the models of higher complexity levels (Figure 4). Since the results of estimating dimensions using DI do not show a clear change in regard to different complexity levels, it is suggested that differences between VR and DI support to spatial perception are more noticeable for the higher levels of complexity. Nevertheless, such propositions should be investigated further, as there might exist other factors that have influenced the results. For instance, participants in VR could use two manipulation strategies, namely rotating the model in their hand (preferred for lower complexity models) or move around the static object (preferred for higher complexity models); while in DI, participants could only rotate the model.

\subsection{Limitations of the study}

There exist limitations which might influence the results of this study and disrupt its validity. The most important is not applying the randomisation of condition order which might give a learning effect to participants for the experiment within the second environment (i.e. VR). Even though some models were changed in-between the two environments to minimise the order effect, future studies should employ randomisation. The second limitation is related to the number of functions in VR, which excluded options such as hiding components and sections cuts. However, when necessary, those operations were conducted by a third party without stopping the review process. Third restraint concerns the limited sample size that diminishes the statistical significance of the results obtained from the collected data. Finally, a homogeneous sample in terms of background, experience and age limits the generalisation of the results. Nonetheless, the exploratory nature of the presented research provides valuable directions for future investigations.

\section{CONCLUSIONS}

The reported study examined differences in spatial perception when reviewing 3D CAD models via VR and DI. The study results show that VR enhances the spatial perception of models at different complexity levels. It has been found that various sensory cues are used to perceive the model size and that the employed sensory cues change with the level of complexity. Participants in DI used UIE cues and familiarity with product's functionality to perceive the model size which was, in terms of dimension estimation and perception of UIE fit, not as insightful as the use of proprioceptive sense and controllers 
in VR. More specifically, in VR more participants could perceive the UIE fit and their dimension estimation had a lower relative error than in DI. Finally, it is proposed that differences between DI and VR for reviewing models are more evident when reviewing models of higher complexity levels. However, future studies are needed to identify sensory cues used for reviewing the models of different complexity, and to unravel the subtle differences between the two investigated technologies.

\section{REFERENCES}

Banerjee, P., Bochenek, G.M. and Ragusa, J.M. (2002), “Analyzing the relationship of presence and immersive tendencies on the conceptual design review process", Journal of Computing and Information Science in Engineering, Vol. 2 No. 1, pp. 59-64. https://doi.org/10.1115/1.1486218

Berg, L.P. and Vance, J.M. (2016), “An industry case study: investigating early design decision making in virtual reality”, Journal of Computing and Information Science in Engineering, Vol. 17 No. 1, p. 011001. https://doi.org/10.1115/1.4034267

Berg, L.P. and Vance, J.M. (2017), "Industry use of virtual reality in product design and manufacturing: a survey”, Virtual Reality, Vol. 21 No. 1, pp. 1-17. https://doi.org/10.1007/s10055-016-0293-9

Chandrasegaran, S.K., Ramani, K., Sriram, R.D., Horváth, I., Bernard, A., Harik, R.F. and Gao, W. (2013), “The evolution, challenges, and future of knowledge representation in product design systems", Computer-Aided Design, Vol. 45 No. 2, pp. 204-228. https://doi.org/10.1016/j.cad.2012.08.006

Coburn, J.Q., Freeman, I. and Salmon, J.L. (2017), "A review of the capabilities of current low-cost virtual reality technology and its potential to enhance the design process", Journal of Computing and Information Science in Engineering, Vol. 17 No. 3, p. 031013. https://doi.org/10.1115/1.4036921

de Casenave, L. and Lugo, J.E. (2017), "Design review using virtual reality enabled CAD”, ASME 2017 International Design Engineering Technical Conferences and Computers and Information in Engineering Conference, Cleveland, August 6-9, p. V001T02A067. https://doi.org/10.1115/DETC2017-67878

Freeman, I., Salmon, J. and Coburn, J. (2018), “A bi-directional interface for improved interaction with engineering models in virtual reality design reviews", International Journal on Interactive Design and Manufacturing, Vol. 12 No. 2, pp. 549-560. https://doi.org/10.1007/s12008-017-0413-0

Gîrbacia, F., Beraru, A., Talabă, D. and Mogan, G. (2012), "Visual depth perception of 3D CAD models in desktop and immersive virtual environments", International Journal of Computers", Communications and Control, Vol. 7 No. 5, pp. 840-848. https://doi.org/10.15837/ijccc.2012.5.1339

Gooch, A.A. and Willemsen, P. (2002), "Evaluating space perception in npr immersive environments", 2nd International Symposium on Non-photorealistic animation and rendering, Annecy, June 3-5, pp. 105-110.

Hou, M. and Hollands, J.G. (2009), "Comparative evaluation of display technologies for collaborative design review”, Presence, Vol. 18 No. 2, pp. 125-138. https://doi.org/10.1162/pres.18.2.125

Hubka, V. and Eder, W.E. (1988), Theory of Technical Systems: A Total Concept Theory for Engineering Design, Springer-Verlag, Berlin Heidelberg, https://doi.org/10.1007/978-3-642-52121-8

Liu, Y., Lather, J. and Messner, J. (2014), "Virtual Reality to Support the Integrated Design Process: A Retrofit Case Study", 2014 International Conference on Computing in Civil and Building Engineering, Orlando, June 23-25, pp. 801-808. https://doi.org/10.1061/9780784413616.100

Mohler, B.J., Di Luca, M. and Bülthoff, H.H. (2013), "Multisensory contributions to spatial perception”, In: Waller, D. and Nadel, L. (Ed.), Handbook of Spatial Cognition, American Psychological Association, Washington, pp. 81-97, https://doi.org/10.1037/13936-005.

Paes, D., Arantes, E. and Irizarry, J. (2017), "Immersive environment for improving the understanding of architectural 3D models: Comparing user spatial perception between immersive and traditional virtual reality systems", Automation in Construction, Vol. 84 No. December, pp. 292-303. https://doi.org/10.1016/j.autcon.2017.09.016

Satter, K. and Butler, A. (2015), "Competitive Usability Analysis of Immersive Virtual Environments in Engineering Design Review", Journal of Computing and Information Science in Engineering, Vol. 15 No. 3, p. 031001. https://doi.org/10.1115/1.4029750

Wolfartsberger, J., Zenisek, J., Sievi, C. and Silmbroth, M. (2017), “A virtual reality supported 3D environment for engineering design review”, 2017 23rd International Conference on Virtual System \& Multimedia (VSMM), Dublin, Oct 31 - Nov 4, IEEE, pp. 1-8. https://doi.org/10.1109/VSMM.2017.8346288

\section{ACKNOWLEDGMENTS}

This paper reports on work funded by the Croatian Science Foundation project IP-2018-01-7269: Team Adaptability for Innovation-Oriented Product Development - TAIDE (http://www.taide.org). The authors would like to thank the students for the participation in the experiment and CADCAM Group for providing the virtual reality equipment. 